\title{
SESSION I
}

\author{
Chairman: DR J. DOMINIAN
}

\section{The neurophysiological aspects of sleep}

\author{
G. W. FENTON \\ F.R.C.P.E., M.R.C.P., M.R.C.Psych. \\ Maudsley Hospital and Institute of Psychiatry, University of London
}

\section{Definition}

Although about a third of our life span is spent in sleep, the sleeping state is difficult to define. It may be regarded as a recurring state of inactivity accompanied by loss of awareness and decrease in responsiveness to the environment. It can be distinguished from coma due to disease, drugs or alcohol, and unconsciousness caused by general anaesthesia, by the ease with which the sleeping subject can be aroused. Stupor, a condition occurring in some mental illnesses and certain brain lesions, is also associated with unresponsiveness and immobility. However, disorder in stupor is one of motility, not consciousness, for the stuporose patient is fully aware of the environment though not responsive to it. Finally, there are a number of conditions of dissociation of consciousness such as hypnotic trances and fugue states, that are intermediate, possessing some of the features of both sleep and wakefulness. In these states, however, the affected individual, although amnesic for his actions, behaves for the most part as if fully in touch with the external world.

\section{Electrophysiological aspects of sleep}

Polygraphic studies (EOG, EMG and EEG) of nocturnal sleep have demonstrated that there is not one, but two opposing states of sleep (Freeman, 1972). One sleep state, non-rapid eye movement (NREM) sleep, contains slow waves, spindles and $\mathrm{K}$-complexes in the EEG recording. No eye movements occur except slow eyeball oscillation during drowsiness and in the lighter stages of NREM sleep. Cardiorespiratory activity is steady with slower heart and respiration rates and during quiet wakefulness. The electrical activity of the chin EMG decreases, but falls further, almost to zero, during the other type of sleep. This second type of sleep, called rapid eye movement sleep (REM), is characterized by a low voltage mixed frequency EEG pattern with bursts of rapid conjugate eye movements (REMs) and profound abolition of tone in the neck muscles. The phenomena in the REM state can be classified into the following two groups: tonic (continuous physiological changes) and phasic (periodic changes). The main tonic events, which persist continuously through the REM state, are the low voltage mixed frequency EEG pattern similar to that accompanying arousal, hippocampal theta rhythm and the abolition of nuchal muscle tone. The phasic phenomena include bursts of rapid eye movements and, in the cat, high voltage spikes, isolated or grouped in volleys that appear in the pons, lateral geniculate nucleus and occipital cortex. These are known as ponto-geniculo-occipital (PGO) spikes. They make their appearance about $0 \cdot 5-1$ min before the occurrence of the tonic phenomenon and subsequently accompany the bursts of REM. The PGO spikes come at a remarkably constant rate; about $60-70$ min, or 1400/day (Jouvet, 1967). The organism is in a state of intense physiological activity during the REM state. Cerebral blood flow is greater than that during wakefulness. Heart rate, respiration and blood pressure become highly variable with higher mean levels. The penis is in a state of tumescence.

When awakened from REM sleep the subject is likely to report vivid dreams with much visual imagery. This contrasts with the mentation reported on waking from NREM sleep, which tends to consist of specific thoughts, devoid of dramatic story content, with minimal sensory imagery, those images that are present being vague, static or fragmentary (Freemon, 1972; Rechtschaffen, 1973). Both REM and NREM mentation slip rapidly from the memory unless rehearsed immediately on reawakening. Hence, a subject may deny having any dreams or interruption with his previous night's sleep, only to 
find to his surprise that he not only had vivid REM mentation but was awakened and gave a detailed dream report (Baekeland and Lasky, 1968).

NREM sleep can be classified into a number of stages according to criteria formulated by a Committee of the Association for the Psychophysiological Study of Sleep (Rechtschaffen and Kales, 1968). These are as follows: stage 1, a relatively low voltage mixed frequency EEG without rapid eye movements; stage 2, 12-14 Hz sleep spindles and $\mathrm{K}$ complexes on a background of relatively low voltage, mixed frequency EEG activity; stage 3, moderate amounts of high amplitude slow wave activity are present with $20-50 \%$ of the epoch consisting of waves of $2 \mathrm{~Hz}$ or less, with a peak-to-peak amplitude of $75 \mu \mathrm{V}$ or more; stage 4, large amounts of high amplitude activity are present, more than $50 \%$ of the epoch consisting of waves of $2 \mathrm{~Hz}$ or less with peak-to-peak amplitude of $75 \mu \mathrm{V}$ or more. Sleep spindles and K-complexes may or may not be present in stages 3 and 4 . Stages 3 and 4 are often referred to as delta wave sleep, or slow wave sleep (SWS).

When a healthy person retires for the night drowsiness develops with increased muscular relaxation, slow eye-ball oscillation and disappearance of the alpha rhythm. Subsequently rapid development of NREM sleep of progressively increasing depth occurs, stage 4 being reached within the first 30-60 min. Gradual slowing of heart and respiration rates, reduction of muscle tone and increasing thresholds of arousal are observed. This sequence of events is interrupted after about $90 \mathrm{~min}$ by the sudden appearance of the REM state with bursts of rapid eye movements, abolition of nuchal muscle tone, skeletal muscular twitches, a variable rapid heart rate, shallow irregular respirations and penile tumescence. The first REM period is brief, usually 5-10 min, with fewer eye movements than later REM periods (Verdone, 1965). Four to six REM periods occur during the night in $80-100$ min cycles, alternating with NREM sleep. Each lasts 10-30 min, the first three REM periods tending to increase progressively in length as the night goes on. Thus, longer periods of REM sleep occur later in the night. In contrast, the bulk of stage 3 and 4 sleep occurs early, while in the latter part of the night NREM sleep consists mainly of stage 2 . In fact, most stage 4 occurs during the first NREM period.

The young adult spends 20 to $25 \%$ of the night in REM, about $5 \%$ in stage 1 , around $50 \%$ in stage 2 , $7-8 \%$ in stage 3 and $10-16 \%$ in stage 4 (Williams, Agnew and Webb, 1964, 1966; Agnew and Webb, $1968 \mathrm{a}$; Kales et al., 1967a, b, c). In the neonate, the sleep cycle is much shorter, $40-45 \mathrm{~min}$, and REM sleep much more abundant, about $50 \%$ of total sleep time (TST). Moreover, sleep onset directly into the REM state may occur and the sleep is dispersed throughout the 24-hr period. The percentage of REM sleep decreases progressively after birth, reaching $20-25 \%$ of TST at the end of the first year. The sleep patterns of children, young adults and elderly differ mainly in total sleep time and stage 4 sleep. Children have considerably more stage 4 sleep $(20-30 \%)$ while this percentage decreases to $10-16 \%$ in young adults with a further dramatic reduction in middle and old age $(0-6 \%)$. The elderly also show a substantial decrease in total sleep time owing to frequent wakenings during the night. REM percentage shows a slight fall to around $20 \%$ in the middle-aged and elderly. While the first three REM periods of children and young adults increase successively in length, the REM periods of the elderly remain fairly uniform in duration (Kales et al., 1967; Feinberg, Koresko and Heller, 1967; Feinberg, 1974). The effects of ageing on sleep are accentuated in elderly patients with diffuse brain pathology.

Most psychoactive drugs also affect REM sleep, the most common response being a varying degree of suppression with a compensatory rebound on withdrawal. The suppressant effect may be temporary, normal REM percentages reappearing with continued administration. Only a few compounds have been shown to increase REM sleep. However, the drug effects on REM sleep are complex and dosedependent. For example, small doses of monoamine oxidase inhibitors (MAOIs) can result in a transiento initial increase in REM time while larger doses cause? marked reduction. Slow wave sleep is also reduced by certain drugs, especially the benzodiazepine derivatives (Oswald, 1973).

\section{Hormone secretion during sleep}

As well as the autonomic changes which occur during sleep, important alterations in endocrine function have been reported in recent years. Sleep is the most important stimulus for human growth hormone (HGH) secretion. Except for increases following hypoglycaemia, exercise and psychic stress, human growth hormone maintains a relatively low constant serum level during wakefulness (Catt, 1970). A peak of growth hormone activity appears shortly after sleep onset and is triggered by the first nightly appearance of slow wave sleep, NREM stages 3 and 4 (Takahaski, Kipnis and Daughaday, 1968; Parker et al., 1969). This physiologically maximal HGH release in slow wave sleep has been taken as evidence that slow wave sleep has an anabolic protein sparing restorative function (Oswald et al., 1973).

The relationship of other pituitary hormones secretion to the sleep waking cycle and to sleep stage is less dramatic. Sassin et al. (1972) have also noted a marked diurnal variation in human plasma prolactin, with highest values during sleep. However, 
periods of episodic release occurred throughout the $24 \mathrm{hr}$. The concentration of ACTH in the plasma cannot be assayed, but the plasma cortisol level is highest in the early morning hours and lowest in the late evening in the human being, with a well established circadian sleep waking cycle. Cortisol begins to be secreted in large episodic bursts during the 3 or $4 \mathrm{hr}$ before time of waking. Although this is also the time of maximum REM activity, there is no clear relationship between REM sleep and cortisol secretion (Weitzman, 1972).

Regarding the pituitary gonadotrophins, Rubin (1972) has described unrelated random peaks of $\mathrm{LH}$ (luteinizing hormone) and FSH (follicle stimulating hormone) during sleep, with the plasma LH being slightly higher in REM sleep in adult males. The observation of a modest but definite increase in plasma LH level in sleep in adult males was confirmed by Rubin et al. (1973) while FSH secretion was found to have no clear relationship to sleep stages. No significant circadian rhythm during sleep for either LH or FSH was apparent. Nankin and Troen (1972) have observed that the pituitary release of $\mathbf{L H}$ occurs as repetitive abrupt discharges in healthy adult men during the night with highest levels in the early morning. Kapen (1972) has studied several females at various phases of the menstrual cycle. During menstruation, the episodic release of LH is infrequent, with low amplitude peaks. Just before ovulation the episodic release of $\mathrm{LH}$ is more prominent, with high amplitude peaks of secretion occurring, half of which occur in REM periods. The secretory episodes also seem to be more frequent with high amplitude peaks in the latter part of the follicular phase. Finkelstein et al. (1973) have reported, that plasma $\mathbf{L H}$ output is synchronized with sleep at the beginning of puberty with the secretion during sleep being greatly augmented during puberty. These workers observed that, when sexual maturation is complete, the rise in LH secretion during sleep is lost. A more recent study from the same laboratory has sampled plasma $\mathrm{LH}$ and testosterone levels at 20-min intervals for $24 \mathrm{hr}$ in nine pubertal boys and three sexually mature young men (Boyar et cl., 1974). In all nine pubertal boys the plasma testosterone concentration fluctuated and was significantly higher during normal nocturnal sleep as compared to daytime waking. This increased testosterone during sleep was temporally linked to the pubertal sleep augmentation of $\mathrm{LH}$ secretion. Reversal of sleep in three of the boys showed that the plasma testosterone concentrations were now augmented during the reversed daytime sleep period. Measurement of the plasma $\mathrm{LH}$ and testosterone levels in the three sexually mature young men showed episodic secretion peaks of $\mathrm{LH}$ and testosterone both during waking and sleep periods. There was no consistent increase of $\mathrm{LH}$ or testosterone secretion during sleep in the young adult males, nor was there any relationship between plasma $\mathrm{LH}$ and testosterone levels, neither during sleep nor wakefulness. Other all-night studies of adult males have reported a decline in plasma testosterone levels with sleep onset, with a subsequent increase in the latter part of the night, reaching a peak in the early morning between 6 and 7 a.m., with many fluctuations, which tend to occur at or before onset of REM periods (Evans et al., 1971; Rowe et al., 1974).

Ban (1972) has reported a diurnal fluctuation in triiodothyronine ${ }^{131} \mathrm{I}$ resin sponge uptake $\left(\mathrm{T}_{3} \mathrm{RSU}\right)$ with its peak during sleep. On the other hand plasma thyroxine concentrations sampled at 20-min intervals throughout the $24 \mathrm{hr}$ have revealed slightly higher levels during wakefulness than during sleep (O'Connor et al., 1974). Disturbance in thyroid function certainly affects slow wave sleep, a marked reduction occurring in hypothyroidism (Kales et al., 1967) and an increased percentage in hyperthyroidism (Dunleavy et al., 1974). The parathyroid hormone shows a maximum output between the hours of 2 and 4 a.m. (Jubiz et al., 1972).

\section{Neural mechanisms of sleep}

The neural mechanisms of sleep and waking are complex and still the subject of much debate. The present position has been elegantly reviewed by Moruzzi (1972). The ascending reticular system and a group of neurones lying in the posterior hypothalamus appear to be endowed with a tonic activating influence and are concerned with the maintenance of wakefulness. In contrast, the lower brainstem (middle and upper middle pons in the region of the solitary tract) and basal forebrain (preoptic region of the anterior hypothalamus) contain structures with an opposing function. These actively inhibit the more rostral portions of the mid-brain reticular formation with consequent synchronization of cortical rhythms and the induction of sleep accompanied by NREM EEG patterns.

The trigger for REM sleep has been located in the pons by a series of coagulation studies in which complete destruction of the nucleus reticularis pontis caudalis has been shown to eliminate REM sleep without affecting NREM sleep. The ascending route from this centre to the cortex has not yet been defined. Bilateral destruction of the locus coeruleus, a pontine nucleus rich in noradrenaline, abolishes the tonic atonia of the REM state due to interference with the descending inhibitory system, which via the reticulo spinal pathways inhibits the spinal anterior horn cells (Jouvet, 1972). The neural basis of the phasic REM components appears to be separate, involving the lateral vestibular nuclei and ocular motor control centres (Pompeiano and Morrison, 1965). 
Neurotransmitters and sleep

Jouvet (1972) has reviewed the role of monoamines and acetylcholine containing neurones in the regulation of sleep. He hypothesizes that the 5hydroxytryptamine (5-HT)-rich neurones in the anterior raphé system* are responsible for behavioural and slow wave sleep. The 5-HT neurones in the caudal raphé nuclei prime REM sleep mechanisms by sending terminals to the caudal two thirds of the locus coeruleus. This in turn is responsible for total inhibition of muscle tone associated with REM. On the other hand the medial third of the locus coeruleus (pontine pace maker) is responsible for both the phasic and tonic ascending REM components. The 5-HT priming mechanisms may trigger the locus coeruleus thus causing the onset of REM while terminals from the locus coeruleus in turn suppress re-uptake of 5-HT thus allowing the REM state to develop. Acetylcholine and noradrenaline are concerned with the maintenance of REM while PGO activity appears to be inhibited by 5-HT. Alterations between slow wave sleep and REM sleep are produced by alternating 5-HT release (SWS) and re-uptake (REM). The anterior noradrenaline-containing cells of the locus coeruleus in the pons cause waking. According to Jouvet, the neurotransmitter involved with the maintenance of wakefulness is acetylcholine.

However, the biochemical findings in recent human sleep studies (Wyatt, 1972) do not readily conform to Jouvet's elegant model of the control of sleep and waking based on animal work. For example, parachlorophenylalanine (PCPA), which decreases brain 5-HT, decreased REM sleep without affecting NREM sleep. 5-Hydroxytryptophan (5HPT), the immediate precursor of 5-HT, reversed the PCPA effect and elevated REM sleep in healthy subjects. The twice removed precursor of 5-HT, tryptophan, had a marked soporific effect in patients with insomnia and increased SWS in normals. Tryptophan also decreased REM sleep in normals. The observation that tryptophan produced the opposite effect on sleep from that of 5-HTP suggests that tryptophan-induced sleep is not caused by its effect on 5-HT metabolism. Other metabolic pathways may be involved. The finding that PCPA enhanced tryptophan's soporific effect is further evidence to support this view.

Thus the hypothesis that 5-HT is primarily involved in initiating and sustaining NREM sleep is not supported by human sleep studies.

In fact, the available evidence points to 5-HT metabolites having a role in triggering the REM

\footnotetext{
* The raphé system is a collection of midline nuclei situated in the brain stem, clustered in nine groups from the nucleus raphé obscurus in the medulla to the nucleus linearis in the mesencephalon.
}

state. Monoamine oxidase inhibitors (MAOIs), which prevent the metabolism of 5-HT, markedly reduce or abolish REM sleep. PCPA blocks the synthesis of 5-HT and reduces REM sleep, an effect which can be reversed by giving 5-HTP, the immediate precursor of 5-HT. These results suggest that interruption of the normal metabolism of 5-HT causes REM reduction and support the 5-HT REM triggering hypothesis. Against this view is the finding that low doses of MAOIs, which should decrease 5-HT metabolites, increase rather than decrease REM sleep. Thus REM reduction is not invariably due to a fall in the brain concentration of 5-HT metabolites.

As hypothesized by Jouvet, the adrenergic system may also play a role in REM control. $\alpha$-Methylparatyrosine (AMPT) and $\alpha$-methyl-phenyl-alanine (AMPA) are both tyrosine hydroxylase inhibitors and decrease the content of brain catecholamines. Both were found to increase REM sleep time, while L-dopa, a catecholamine precursor, decreased REM sleep. Thus, in man, it appears that high brain catecholamine concentrations decrease REM, while lowering catecholamine levels augments REM.

Wyatt (1972) claims that these findings indicate that the REM state is controlled by an interaction between the serotonergic and adrenergic systems $?$ with 5-HT augmenting and catecholamines reducinge the REM state. There is considerable biochemicalo evidence that catecholamines can modify $5-\mathrm{HT} ?$ metabolism.

\section{Humoral factors}

Humoral factors may play a role in the genesis of sleep. Recipient rabbits infused with a dialysate from the cerebral sinuses of sleeping donor rabbits (sleep being induced by stimulation of the ventromedial intralaminar thalamic nuclei) developed SWS (Monnier and Hatt, 1971; Monnier et al., 1972). Fencl, Koki and Pappenheimer (1971) have also isolated a factor derived from sleep-deprived goat C.S.F. which increases sleep time and decreases locomotor activity in rats.

\section{References}

Agnew, H.W. \& WeBb, W.B. (1968) Sleep patterns of 30 to 39 year old male subjects (abstract). Psychophysiology, 5 , 228.

BAEKeland, F. \& LASKY, R. (1968) The morning recall of rapid eye movement period reports given earlier in the night. Journal of Nervous and Mental Disease, 147, 570.

BAN, Y. (1972) A study of the diurnal variation in the level of serum thyroid hormone. Folia encocrinologica iaponica, 48, 268.

Boyar, R., Perlow, M., Hellman, L., Kapen, S. \& WeITzMAN, E. (1972) Twenty-four hour pattern of luteinizing hormone secretion in normal men with sleep stage recording. Journal of Clinical Endocrinology and Metabolism, 35, 73. 
Boyar, R.M., Rosenfeld, R.S., Kapen, S., Finkelstein, J.R., Roffwarg, R., Weitzman, E.D. \& Hellman, L. (1974) Human puberty: simultaneous augmented secretion of luteinizing hormone and testosterone during sleep. Journal of Clinical Investigation, 54, 609.

CATT, K.J. (1970) Growth hormone. Lancet, i, 933.

Dunleavy, D.L.F., Oswald, I., Brown, P. \& Strong, J.A. (1974) Hyperthyroidism, sleep and growth hormone. Electroencephalography and Clinical Neurophysiology, 36, 259.

Evans, J.I., Maclean, A.M., Ismail, A.A.A. \& Love, D. (1971) Circulating levels of plasma testosterone during sleep. Proceedings of the Royal Society of Medicine, 64, 31 .

Feinberg, I., Koresko, R.J. \& Heller, N. (1967) EEG sleep patterns as a function of normal and pathological ageing in man. Journal of Psychiatric Research, 5, 107.

FeINBERG, I. (1974) Changes in sleep cycle alterations with age. Journal of Psychiatric Research, 10, 283.

FenCl, V., KoKi, G. \& PAPPenheimer, J.R. (1971) Factors in cerebrospinal fluid from goat that affect sleep and activity in rats. Journal of Physiology. London and Cambridge, 261, 565 .

Finkelstein, J.W., Boyar, R.M., Roffwarg, H. \& Hellman, L. (1973) Synchronization of luteinizing hormone secretion with sleep at the initiation of puberty. Acta paediatrica scandinavica, 62, 92.

Freemon, F.R. (1972) Sleep Research. A Critical Review. Thomas, Springfield, Illinois.

Jouver, M. (1967) Neurophysiology of the states of sleep. Physiological Reviews, 47, 117.

Jouvet, M. (1972) The role of monoamines and acetylcholine-containing neurones in the regulation of the sleep waking cycle. Ergebnisse der Physiologie, 64, 166.

Jubiz, W., Canterbury, J.M., Reiss, E. \& Tyler, F.H. (1972) Circadian rhythm in serum parathyroid hormone concentration in human subjects: correlation between serum calcium, phosphate, albumin and growth hormone levels. Journal of Clinical Investigation, 51, 204.

Kales, A., Henser, G., Jacobson, A., Kales, J.D., Hanley, J., Zweizig, J.R. \& PAUlson, M.J. (1967a) All-night sleep studies in hypothyroid patients, before and after treatment. Journal of Clinical Endocrinology and Metabolism, 27, 1593.

Kales, A., Jacobson, A., Kales, J.D., Kun, T. \& Weissbuch, R. (1967b) All-night EEG sleep measurements in young adults. Psychonomic Science, 7, 67.

Kales, A., Wilson, T., Kales, J.D., Jacobson, A., Paulson, M.J., Kollar, E. \& Walter, R.D. (1967c) Measurements of all night sleep in normal elderly persons: effects of ageing. Journal of the American Geriatrics Society, 15, 405.

KAPEN, D. (1972) Periodicity in sleep and waking states In: The Sleeping Brain (Ed. by M. H. Chase) p. 231. Brain Information Service/Brain Research Institute, University of California Press.

MonNier, M. \& HATt, A.M. (1971) Humoral transmission of sleep. V. New evidence from production of pure sleep haemodialysate. Pflügers Archiv für die gesamte Physiologie des Menschen und der Tiere, 329, 231.

Monnier, M., Hatt, A.M., Cueni, L.B. \& Schoenenberger, G.A. (1972) Humoral transmission of sleep. VI. Purification and assessment of a hypnogenic fraction of 'sleep dialysate' (Factor delta). Pflügers Archiv für die gesamte Physiologie des Menschen und der Tiere, 331, 257.
MoruzzI, G. (1972) The sleep-waking cycle. Ergebnisse der Physiologie, 64, 2.

NANkIN, H.R. \& Troen, P. (1972) Overnight patterns of serum luteinizing hormone in normal men. Journal of Clinical Endocrinology and Metabolism, 35, 705.

O'Connor, J.F., Wu, G.Y., Gallagher, T.F. \& Hellman, L. (1974) The 24-hour plasma thyroxin profile in normal man. Journal of Clinical Endocrinology and Metabolism, 39, 790.

Oswald, I. (1973) Drug research and human sleep. Annual Review of Pharmacology, 13, 243.

Oswald, I., Dunleavy, D.L.F., Allen, S. \& Lewis, S.A. (1973) Neurochemical studies of sleep. In: The Nature of Sleep (Ed. by U. J. Jovanović) p. 280. Fischer, Stuttgart.

Parker, D.C., Sassin, J.F., Mace, J.W., Gotlin, R.W. \& Rossman, L.G. (1969) Human growth hormone release during sleep: electroencephalographic correlation. Journal of Clinical Endocrinology and Metabolism, 29, 871.

Pompeiano, O. \& Morrison, A. (1965) Vestibular influences during sleep: I. Abolition of the rapid eye movements of desynchronized sleep following vestibular lesions. Archives italiennes de biologie, 103, 569.

RECHTSCHAFFEN, A. (1973) The psychophysiology of mental activity during sleep. In: The Psychophysiology of Thinking (Ed. by F. J. McGuigan \& R. A. Schoonover) p. 153. Academic Press, New York and London.

RechtschafFen, A. \& Kales, A. (1968) A manual of standardised terminology, techniques and scoring systems for sleep stages of hunam subjects. Brain Information Service/Brain Research Institute, University of California Press.

Rowe, P.H., Lincoln, G.A., Racey, P.A., Lehane, J., Stephenson, M.J., Shenton, J.C. \& Glover, T.D. (1974) Temporal variations of testosterone levels in the peripheral blood plasma of men. Journal of Endocrinology, 61, 63.

Rubin, R.T. (1972) Periodicity in sleep and waking states. In: The sleeping Brain (Ed. by M. H. Chase) p. 228. Brain Information Service/Brain Research Institute, University of California Press.

Rubin, R.T., Gouin, P.R., Kales, A. \& Odell, W.D. (1973) Luteinizing hormone, follicle stimulating hormone and growth hormone secretion in normal adult men during sleep and dreaming. Psychosomatic Medicine, 35, 309.

Sassin, J.F., Frantz, A.C., Weitzman, E.D. \& Kapen, S. (1972) Human prolactin: 24-hour pattern with increased release during sleep. Science, 177, 1205.

TAKaHASKI, Y., KIPNIS, D.M. \& DaUghadaY, W.H. (1968) Growth hormone secretion during sleep. Journal of Clinical Investigation, 47, 2079.

Verdone, P. (1965) Temporal reference of manifest dream content. Perceptual and Motor Skills, 20, 1253.

Weitzman, E.D. (1972) Periodicity in sleep and waking states. In: The Sleeping Brain (Ed. by M. H. Chase) p. 214. Brain Information Service/Brain Research Institute, University of California Press.

Williams, R.L., Agnew, H.W. \& Webb, W.B. (1964) Sleep patterns in young adults: an EEG study. Electroencephalography and Clinical Neurophysiology, 17, 376.

Williams, R.L., Agnew, H.W. \& WebB, W.B. (1966) Sleep patterns in young females: an EEG study. Electroencephalography and Clinical Neurophysiology, 20, 264.

WyATT, R.J. (1972) The serotonin-catecholamine-dream bicycle: a clinical study. Biological Psychiatry. 5, 33. 